\title{
Measurement based Human Brain Tumor Recognition by Adapting Support Vector Machine
}

\author{
Chandrakant Biradar, Shantkumari \\ ${ }^{I}$ (Computer Science, Poojya Doddappa Appa College Of Engineering \& Technology Gulbarga/ Visvesvaraya \\ Technology University Belgaum, India) \\ ${ }_{2}^{2}$ (Computer Science, Poojya Doddappa Appa College Of Engineering \& Technology Gulbarga/ Visvesvaraya \\ Technology University Belgaum, India )
}

\begin{abstract}
In this work we have proposed a fully automatic algorithm to detect brain tumors by using digital image processing techniques is proposed. Here we detect the tumor, segment the tumor and calculate the area of the tumor. The complex problem of segmenting tumor in MRI can be successfully addressed by considering multi-step approaches. The tumor detection is often an essential preliminary phase to solve the segmentation problem successfully. The experiments showed good results also in complex situations. Segmentation of images embraces a significant position in the region of image processing. Tumor segmentation and area calculation from MRI data is an essential but fatigue, boring and time unbearable task when it completed manually by medical professional when evaluate with present day's high speed computing machines which facilitate us to visual study the area and position of unnecessary tissues. We use SVM classifier to classify the type of tumor.
\end{abstract}

Keywords: - MRI image, DWT, Segmentation, Feature extraction, SVM, Tumor detection.

\section{INTRODUCTION}

The principle of our task is to recognize a tumor and its quantifications from a particular MRI scan of a brain image using digital image processing techniques and compute the area of the tumor by fully automated process and its symmetry analysis. In recent years a great effort of the research in field of medical imaging was focused on brain tumors segmentation. The automatic segmentation has great potential in clinical medicine by freeing physicians from the burden of manual labeling whereas only a quantitative measurement allows to track and modeling precisely the disease. Despite the undisputed usefulness of automatic tumor segmentation, this is not yet a widespread clinical practice, therefore the automatic brain tumor segmentation is still a widely studied research topic. The main difficulties in field of automatic tumor segmentation are related to the fact that the brain tumors are very heterogeneous in terms of shape, color, texture and position and they often deform other nearby anatomical structures. An healthy brain has a strong sagittal symmetry, that is weakened by the presence of tumor. The comparison between the healthy and ill hemisphere, considering that tumors are generally not symmetrically placed in both hemispheres, was used to detect the anomaly. One such artifact is the additional cranial tissues (skull). These additional cranial tissues repeatedly hamper with the ordinary tissues throughout segmentation that accounts for the substandard segmentation efficiency. Magnetic Resonance Imaging (MRI) is an advanced medical imaging technique used to produce high resolution images of the parts contained in the human body.MRI imaging is often used when treating brain tumors. These high resolution images are used to examine human brain development and discover abnormalities. Nowadays there are several methodologies for classifying MR images. Among all medical image processing, image segmentation is initial and important work, for example, quantification of specified area must based on accurate segmentation.

An brain tumor is an intracranial mass produced by an uncontrolled growth of cells either normally found in the brain such as neurons, lymphatic tissue, glial cells, blood vessels, pituitary and pineal gland, skull, or spread from cancers primarily located in other organs [1]. Brain tumors are classified based on the type of tissue involved, the location of the tumor, whether it is benign or malignant, and other considerations. Primary (true) brain tumors are the tumors that originated in the brain and are named for the cell types from which they originated. They can be benign (non cancerous), meaning that they do not increase in a different place or attack neighboring tissues.

They can also be malignant and invasive (spreading to neighboring area). Secondary or metastasis brain tumors take their origin from tumor cells which increase to the brain from a different position in the body. Most frequently cancers that increase to the brain to reason secondary brain tumors begin in the lumy, breast, and kidney or from melanomas in the skin. The first aim of this work is to develop a framework for a robust and accurate segmentation of a large class of brain tumors in MR images. Most existing methods are region-based. They have several advantages, but line and edge information in computer vision systems are also important. The proposed method tries to combine region and edge information, thus taking advantage of both approaches while 
cancelling their drawbacks. We first segment the brain to remove non-brain data. However, in pathological cases, standard segmentation methods fail, in particular when the tumor is located very close to the brain surface. Therefore we propose an improved segmentation method, relying on the approximate symmetry plane.

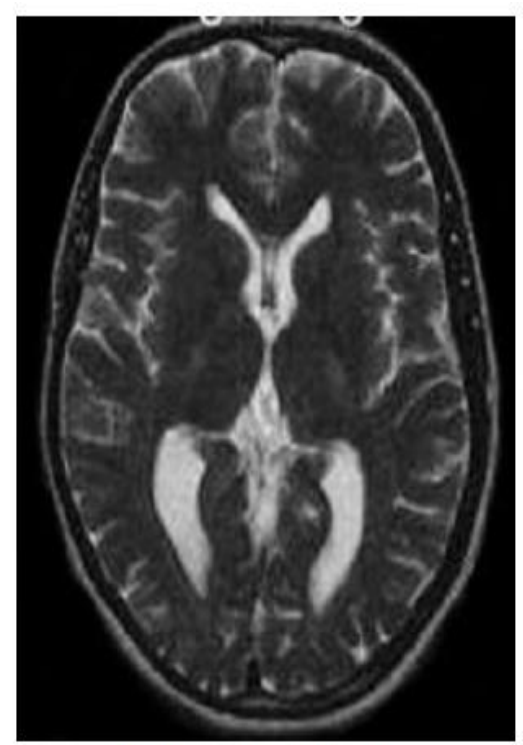

(a) normal brain

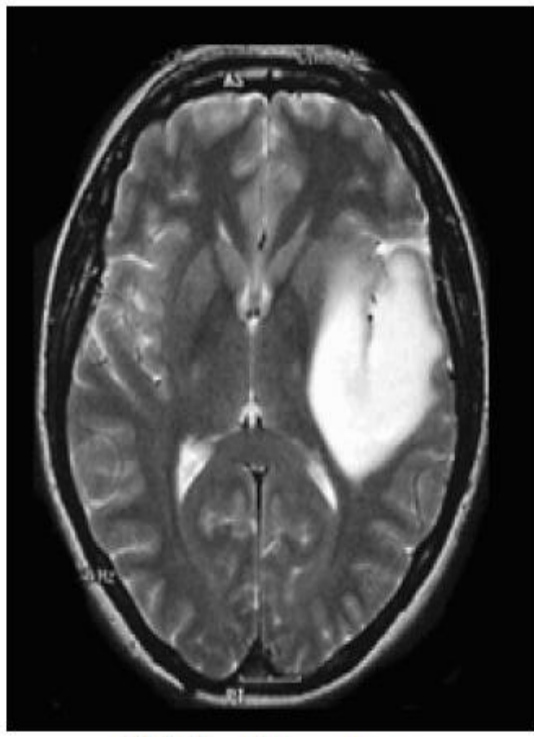

(b) benign tumor

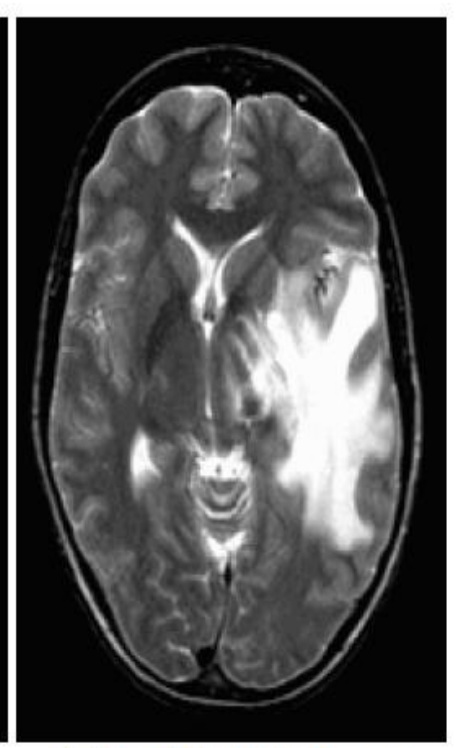

(c) malignant tumor

\section{RELATEDWORK}

Image segmentation represents a method of separation a portion of image into separate area. A great assortment of dissimilar segmentation approaches for images have been developed. The Segmentation of an image is the division or separation of the image into regions of similar attribute. The ultimate aim in a large number of image processing applications is to extract important features from the image data, from which a understanding of the scene can be provided by the machine. Among them, the clustering technique have been comprehensively explore and used in T.Logeswari and M.Karnan [2], a clustering support come close to using a self organizing map (SOM) algorithm is projected for medical image segmentation. This paper illustrate segmentation scheme consists of two stages. In the opening stages, the MRI brain image is obtained from patient database. In that film artifact and noise are disconnected. In the subsequent stages (MR) image segmentation is to precisely recognize the major tissue arrangement in these image areas. In R. Rajeswari et al. [3] proposed a Spectral leakage has the effect of the frequency analysis of finite-length signals or finite-length segments of infinite signals. In brain the tumor itself, comprising a necrotic (dead) part and an active part, the swelling in the nearby brain, As all tumor do not have a clear boundary between active and necrotic parts there is need to define a clear boundary between edema and brain tissues. Hassan Khotanlou et all [4] recommend a common automatic scheme for segmenting brain tumors in 3D MRI.

Our scheme is valid in dissimilar types of tumors with MRI images. Its effect represent the initialization of a segmentation technique based on a mixture of a deformable model and spatial associations, principal to a particular segmentation of the tumors. P.Narendran, V.K. Narendira Kumar, K. Somasundaram [5] proposed a new method for segmentation of pathological brain structures. This method combines prior information of structures and image information (region and edge) for segmentation. The automated brain tumor segmentation method that we have developed consists of two main components: pre processing and segmentation. The inputs of this system are two different modalities of MR images: CE-T1w and FLAIR that we believe are sufficient for brain tumor segmentation [6]. The Graph Cut [7] method attempts to solve the min cut/max flow problem. Snakes and Level Sets are active contour methods that evolve a curve based upon geometric and image constraints. For the problem of brain tumor segmentation, Lefohn et al. [8] implemented a level set solver on the GPU. Quantitative results of this level set formulation compare well with hand contouring results. Kaus et al. [9] used an atlas and statistical information to segment brain tumors. Edward Kim et al. [10] method utilizes statistical seed distributions to overcome the local bias seen in the traditional cellular automata framework. Our results show improved accuracy, robustness, and competitive usability. 


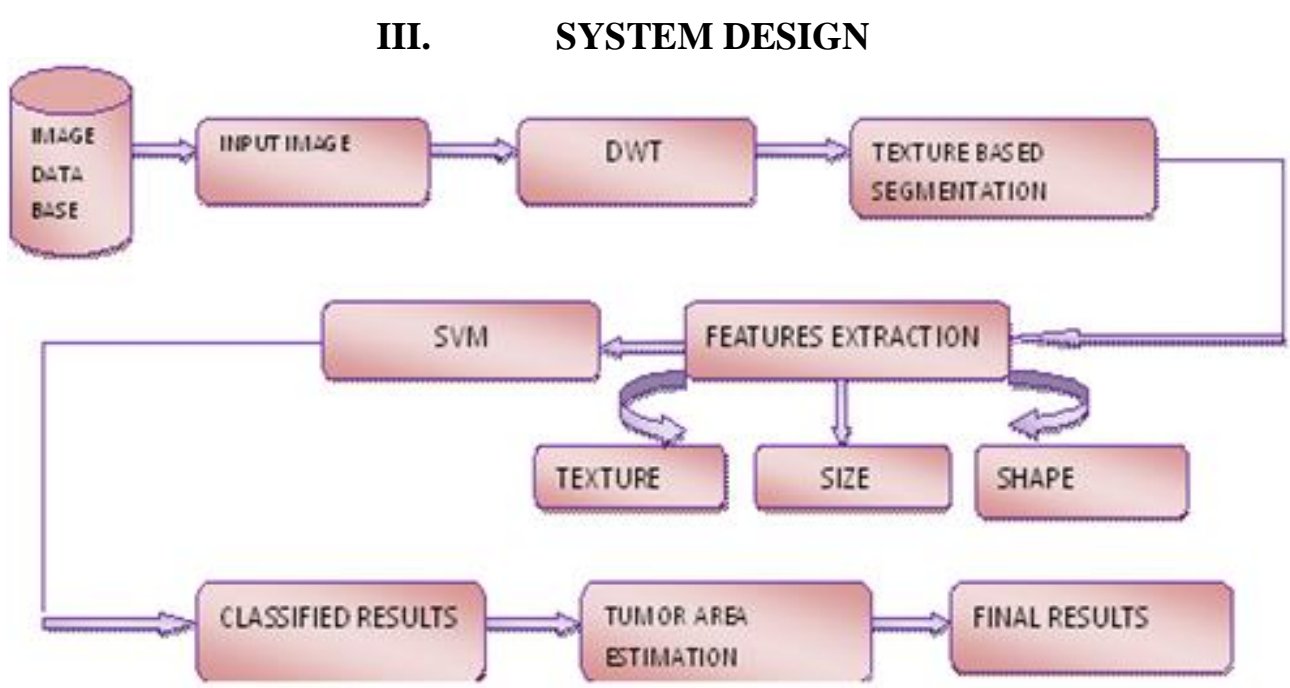

Fig 1 :- System Architecture

Input images are collected from the hospital, net. will apply the DWT on the collected images to decompose an image repeatedly in the low frequency channel which consists of maximum information about the image then we apply the segmentation on the DWT image to extract ROI region by using GLCM as shown in the fig 1 . After the segmentation we extract the features from the segmented image features are texture, size and shape features are extracted. Obtained three features will be used for the classification to classify the type of tumor.

\section{IV.I DWT}

\section{MATERIALS AND METHODS}

The Continuous Wavelet Transform (CWT) is provided by equation 2.1, where $\mathrm{x}(\mathrm{t})$ is the signal to be analyzed. $\psi(t)$ is the mother wavelet or the basis function. All the wavelet functions used in the transformation are derived from the mother wavelet through translation (shifting) and scaling (dilation or compression).

$$
X_{W T}(\tau, s)=\frac{1}{\sqrt{|s|}} \int x(t) \cdot \psi^{*}\left(\frac{t-\tau}{s}\right) d t
$$

The mother wavelet used to generate all the basis functions is designed based on some desired characteristics associated with that function. The translation parameter $\tau$ relates to the location of the wavelet function as it is shifted through the signal. Thus, it corresponds to the time information in the Wavelet Transform. The scale parameter $s$ is defined as $\mid 1 /$ frequency| and corresponds to frequency information. Scaling either dilates (expands) or compresses a signal. Large scales (low frequencies) dilate the signal and provide detailed information hidden in the signal, while small scales (high frequencies) compress the signal and provide global information about the signal.

The following snapshot :(a) consists of two images of which one is input image and the other image we get after applying DWT

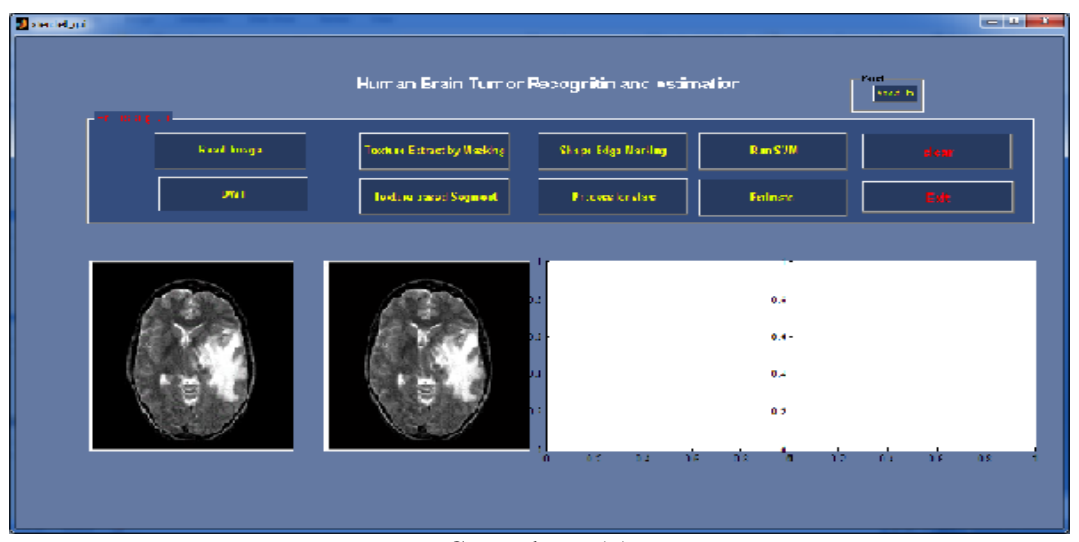

Snapshot :(a) 


\section{IV.II Texture Extraction}

Texture is an important characteristic of the image, describing the spatial pattern of the neighboring pixels' intensities, and is commonly used in image segmentation. A multi-channel filtering approach using a bank of 3D Gabor filters with different orientations and spatial frequencies is employed to extract texture feature vectors from each MRI.

The following snapshot: (b)shows the image after extracting the texture.

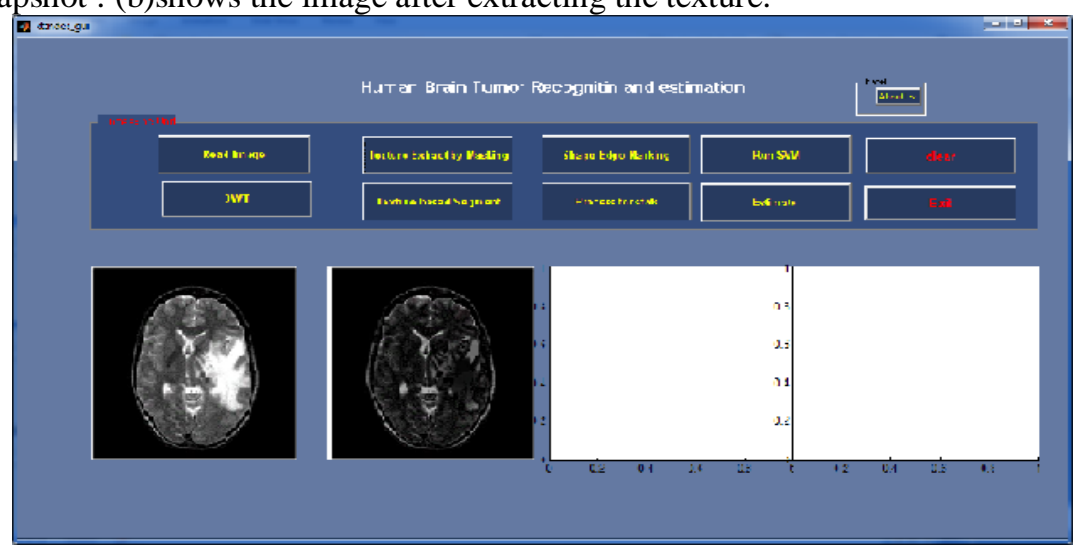

Snapshot : (b)

The GLCM is used for texture extraction. It contains important information about the texture in the examined area of the image. The gray level co-occurrence matrix (GLCM) is just the tool to start and then get the indicators:

1. Skewness : $e^{x}=\frac{E(x-\mu)^{3}}{\sigma^{3}}$

where $\mu$ is the mean of $\mathrm{x}, \sigma$ is the standard deviation of $\mathrm{x}$, and $\mathrm{E}(\mathrm{t})$ represents the expected value of the quantity t.

2. Kurtosis : $e^{x}=\frac{E(x-b)^{4}}{\sigma^{4}}$

where $\mu$ is the mean of $x, \sigma$ is the standard deviation of $x$, and $E(t)$ represents the expected value of the quantity t.

The values are grouped separately depends on the matching pixels and classify the type of tumor.

\section{IV.III Feature Extraction}

Feature extraction involves simplifying the amount of resources required to describe a large set of data accurately. When performing analysis of complex data one of the major problems stems from the number of variables involved. Analysis with a large number of variables generally requires a large amount of memory and computation power or a classification algorithm which over fits the training sample and generalizes poorly to new samples. Feature extraction is a general term for methods of constructing combinations of the variables to get around these problems while still describing the data with sufficient accuracy. In image processing, feature extraction is a special form of dimensionality reduction. When the input data to an algorithm is too large to be processed and it is suspected to be notoriously redundant then the input data will be transformed into a reduced representation set of features.

The following snapshot: (c)shows the feature extraction

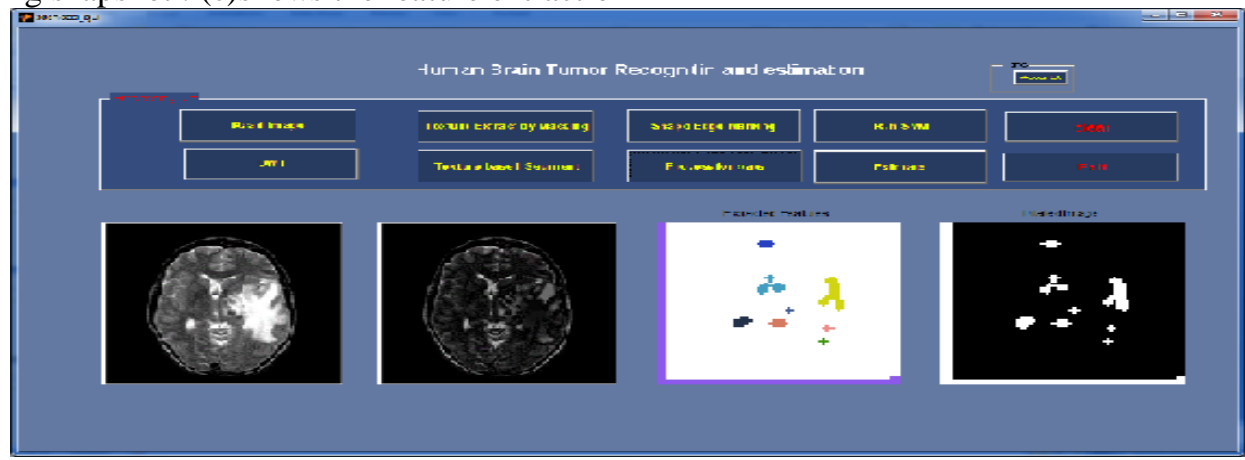

Snapshot : (c) 
Area:

To calculate the area of an irregular figure, follow these steps:

1. Divide the irregular figure into regular figures.

2. Look for missing measurements that you will need to find the area of each new regular figure.

3. Find the area of every regular figure.

4. Add the areas of each regular figure together to find the total area.

1. Diameter : $\mathrm{D}=2 \mathrm{R}$

where $r$ is radius

Once a feature selection finds a proper representation, a classifier can be designed using a number of possible approaches. The performance of classifier depends on the interrelationship between sample size, number of features and classifier(s) complexity. The choice of a classifier(s) is a difficult problem!!! It is often based on which classifier(s) happen to be available or best known to the user.

SVM

Support Vector Machine (SVM) is a powerful supervised classifier and accurate learning technique that has been introduced in 1995. It is derived from the statistical theory developed by Vapnick in 1982 . It yields successful classification results in various application domains, e.g. medical diagnosis . Support Vector Machine (SVM) is based on the structural risk minimization principle from the statistical learning theory. Its kernel is to control the empirical risk and classification capacity in order to maximize the margin between the classes and minimize the true costs . A support vector machine searchs an optimal separating hyper-plane between members and non-members of a given class in a high dimension feature space.

SVM is a binary classifier based on supervised learning which gives better performance than other classifiers. SVM classifies between two classes by constructing a hyperplane in high-dimensional feature space which can be used for classification. Hyperplane can be represented by equation-

$\mathrm{w} \cdot \mathrm{x}+\mathrm{b}=0$

$\mathrm{W}$ is weight vector and normal to hyper plane, $\mathrm{b}$ is bias or threshold.

The following snapshot: (d)shows the result of the system

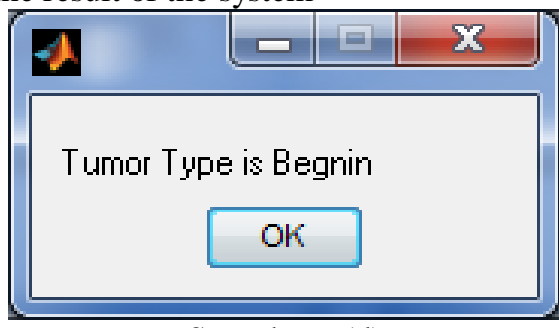

Snapshot : (d)

\section{CONCLUSION AND FUTURE WORK}

We proposed an interactive segmentation method that enables users too quickly and efficiently segment tumors in MRI of brain. We proposed a new method that in addition to area of the region and edge information uses a type of prior information, Since tumor is a rather general concept in medicine, limitations of the proposed approach might become apparent as soon as unforeseen pathologic tissue types that could not adequately be captured by the discriminative model appear in previously unseen patients. Especially secondary tumors might be composed of an enormous variety of tissue types depending on the primary tumor site. Its application to several datasets with different tumors sizes, intensities and locations shows that it can automatically detect and segment very different types of brain tumors with a good quality.

For our future work, we plan to work with a greater number of brain structures and explore incorporating additional information to guide our proposal. We would also like to explore higher dimensional data and improve our user interface and investigate possibilities to handle this issue. The goal is to detect, to segment, and to identify most types of pathological tissue that occur within pediatric brain tumors.

\section{REFERENCES}

[1] Dou, W., Ruan, S., Chen, Y., Bloyet, D., and Constans, J. M. (2007), “A framework of fuzzy information fusion for segmentation of brain tumor tissues on MR images”, Image and Vision Computing, 25:164171.

[2] T.Logeswari and M.Karnan, "An Improved Implementation of Brain Tumor Detection Using Segmentation Based on Hierarchical Self Organizing Map", International Journal of Computer Theory and Engineering, Vol. 2, No. 4, August, 2010,pp.1793-8201. 
[3] R. Rajeswari and P. Anandhakumar, "Segmentation and Identification of Brain Tumor MRI Image with Radix4 FFT Techniques", European Journal of Scientific Research, Vol.52 No.1 (2011), pp.100-109.

[4] Hassan Khotanlou, Olivier Colliot and Isabelle Bloch, "Automatic brain tumor segmentation using symmetry analysis and deformable models", GET-Ecole Nationale Superieure des Telecommunications, France.

[5] P.Narendran, V.K. Narendira Kumar, K. Somasundaram, "3D Brain Tumors and Internal Brain Structures Segmentation in MR Images", I.J. Image, Graphics and Signal Processing, 2012, 1, 35-43.

[6] Dou, W., Ruan, S., Chen, Y., Bloyet, D., and Constans, J. M. (2007), “ A framework of fuzzy information fusion for segmentation of brain tumor tissues on MR images", Image and Vision Computing, 25:164171.

[7] Y. Boykov and V. Kolmogorov. An experimental comparison of min-cut/max-flow algorithms for energy minimization in vision. IEEE Transactions on Pattern Analysis and Machine Intelligence, pages 1124-1137, 2004.

[8] A. Lefohn, J. Cates, and R. Whitaker. Interactive, gpu- based level sets for $3 d$ segmentation. MICCAI, pages 564-572, 2003.

[9] M. Kaus, S. K. Warfield, A. Nabavi, P. M. Black, F. A. Jolesz, and R. Kikinis. "Automated Segmentation of MRI of Brain Tumors", Radiology, 218(2)(586-91), 2001 Feb.

[10] Edward Kim, Tian Shen, Xiaolei Huang, "A Parallel Cellular Automata with Label Priors for Interactive Brain Tumor Segmentation", Lehigh University, Department of Computer Science and Engineering, Bethlehem, PA, USA, 2010.

[11] Sudipta Roy and Prof. Samir K. Bandyopadhyay "Contour Detection of Human Knee", IJCSET ,September 2011, Vol 1, Issue 8,pp. 484-487.

[12] T. Logeswari and M. Karnan , "An improved implementation of brain tumor detection using segmentation based on soft computing" Journal of Cancer Research and Experimental Oncology Vol. 2(1) pp. 006-014, March, 2010.

[13] Prof. Samir K. Bandyopadhyay and Sudipta Roy "Detection of Sharp Contour of the element of the WBC and Segmentation of two leading elements like Nucleus and Cytoplasm", International Journal of Engineering Research and Applications (IJERA), Vol. 2, Issue 1,JanFeb 2012, pp.545-551.

[14] J. J. Corso, E. Sharon, and A. Yuille, "Multilevel Segmentation and Integrated Bayesian Model Classification with an Application to Brain Tumor Segmentation," in Medical Image Computing and Computer Assisted Intervention, vol. 2, 2006, pp. 790-798.

[15] Sudipta Roy and Prof. Samir K. Bandyopadhyay "Visual Image Based Hand Recognitions", Asian Journal Of Computer Science And Information Technology1:4 (2011), pp.106 - 110. 